\title{
The Effects of Spoilers on Jet Mixing of Lobed Nozzles
}

\author{
Liu Dawei1, Huang Jun¹, Sheng Zhiqiang ${ }^{1}$, Ji Jinzu¹
}

\begin{abstract}
In this study, spoilers were installed on the lobed nozzle principal model. Keeping axial position unchanged, the different widths of spoilers were mounted along the circumferential direction of a mixer tube. The effects of spoilers on the jet mixing of lobed nozzle were investigated by numerical method, and the results showed that, before or after the installation, the flow ratio did not change significantly. The value of thermal mixing efficiency and total pressure recovery coefficient had a corresponding improvement. The spoilers increased the borderline area of the primary and secondary streams, thus the mixing was accelerated. Spoilers can avoid high-temperature primary stream impinging the wall of the tube, and the temperature of mixing tube wall decreased significantly.
\end{abstract}

KEYWORDS: Spoiler, Jet mixing, Lobed nozzle, Streamwise vortices, Numerical simulation.

\section{INTRODUCTION}

Because of the especial shape, lobed nozzles can mix the primary and secondary streams with high effectiveness and induce lower pressure loss (Shumpert 1980). Investigations concluded that the increase in the interfacial area between the primary and secondary streams (Elliott et al. 1992) - at the same time forming inviscid streamwise vortices at the wake (Povinelli and Anderson 1984) - and the streamwise vortices interacting with the normal vortices ring which are formed at the interface of the streams for the gradient of the axial velocity (McCormick and Bennett 1994), are the dominating factors for high mixing effectiveness. The lobed nozzles have been widely used in the engineering applications (Presz et al. 1994; Acheson et al. 2011). For instance, the lobed nozzles are widely used for the helicopter or warship infrared suppressor to pump cool air and mix it with the engine exhaust gas to suppress infrared radiation (Pan et al. 2013a; Pan et al. 2014).

In the practical applications of the helicopters, the mixing distance of the infrared suppresser imbedded in the fuselage as a whole is generally short. In order to improve the mixing efficiency in a short mixing distance, large lobe penetration angles are generally adopted. Large lobe penetration angle lobe may cause the primary stream impinging the mixer tube walls, then the temperature and the pressure loss increase (O'Sullivan et al.; 1996; Shan and Zhang 2005; Pan et al. 2013b). The installation of spoilers may be an efficient method to solve this problem. In previous investigations, Sheng et al. (2015b) installed 2 types of spoilers at the lobe peaks and analyzed the influence and mechanisms of spoilers on the lobed nozzles jet mixing. The authors found that the spoilers installed at the lobe peaks can

1.Beijing University of Aeronautics and Astronautics - School of Aeronautic Science and Engineering - Department of Aircraft Design - Beijing - China.

Author for correspondence: Ji Jinzu | Beijing University of Aeronautics and Astronautics - School of Aeronautic Science and Engineering - Department of Aircraft Design | 37 Xueyuan Rd, Haidian | Postal code: 100191 - Beijing - China | Email: jijinzu@buaa.edu.cn

Received: 01/24/2016 | Accepted: 06/20/2016 
induce new streamwise vortices, transform the distribution of the primary stream, then accelerate the mixing in the region off the lobe peaks which can be effectively utilized to lower the temperature of the mixing stream near the duct. This has a dual effect on the suppression of the infrared radiation of the plume and wall (Sheng et al. 2015b).

In this paper, we produced different spoiler lobed nozzles schemes by mounting different widths spoilers inside the mixer tube. In order to simulate the downwash, the secondary flow was also set to velocity inlet, and the velocity was set to $25 \mathrm{~m} \cdot \mathrm{s}^{-1}$. Then the effects of jet mixing and the changes of flow-field were analyzed.

\section{GEOMETRICAL CONFIGURATIONS}

Figure 1 shows the jet mixing model, wherein the nozzle is baseline lobed nozzles (BLN). The annular entrance of the nozzle is formed by 2 circles of diameters 210 and $400 \mathrm{~mm}$, respectively. Through a cone of length equal to $262.5 \mathrm{~mm}$, the annular section is smoothly transformed into a circular one. The lobes are $44 \mathrm{~mm}$ wide and the inward and outward lobe penetration angles are $23.15^{\circ}$ and $21.85^{\circ}$, respectively. The diameters of the circles at the lobe peaks and troughs are, respectively, 550 and $240 \mathrm{~mm}$. The distance from the exit to the entrance is $600 \mathrm{~mm}$, and the exit has an equivalent diameter $d$ of $400 \mathrm{~mm}$. The mixing duct is $1,150 \mathrm{~mm}$ long and its entrance is $100 \mathrm{~mm}$ ahead of the exit of the nozzle. The diameter $D$ of the duct is $700 \mathrm{~mm}$, which gives a length-to-diameter ratio of the mixing segment $L / D=1.5$.

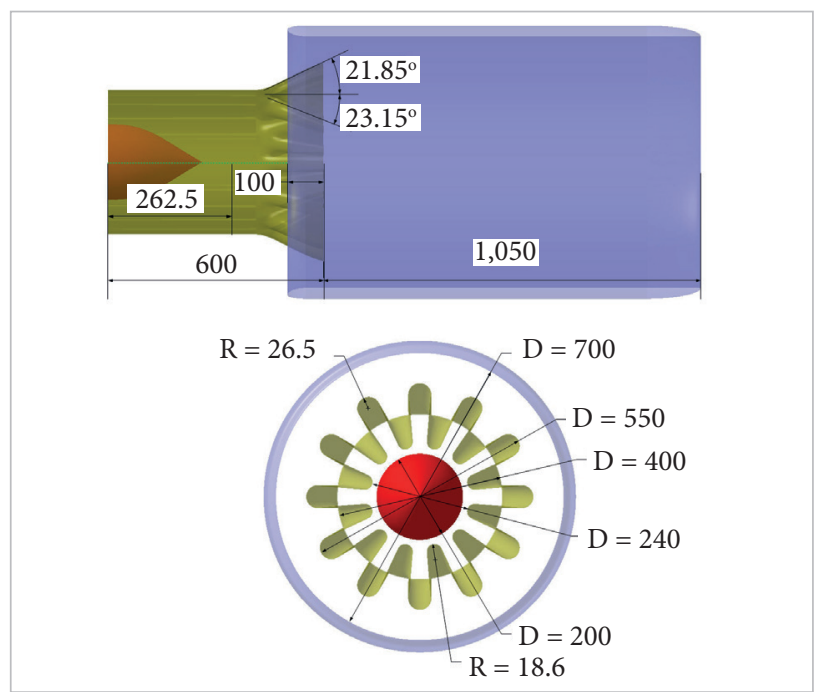

Figure 1. Geometrical dimensions of the base lobe mixer.
Figure $2 \mathrm{a}$ shows the spoiler lobed nozzles which are investigated in this paper; the geometrical dimensions for the spoiler are shown in Fig. 2b. The spoiler lobed nozzles 1, 2 and 3 (S1, S2 and S3) have the same axial length, but with the width and angle increased from $S 1$ to $S 3$. The angles of the spoiler $\theta$ for $\mathrm{S} 1, \mathrm{~S} 2$ and $\mathrm{S} 3$ are $40^{\circ}, 60^{\circ}$ and $90^{\circ}$, respectively.

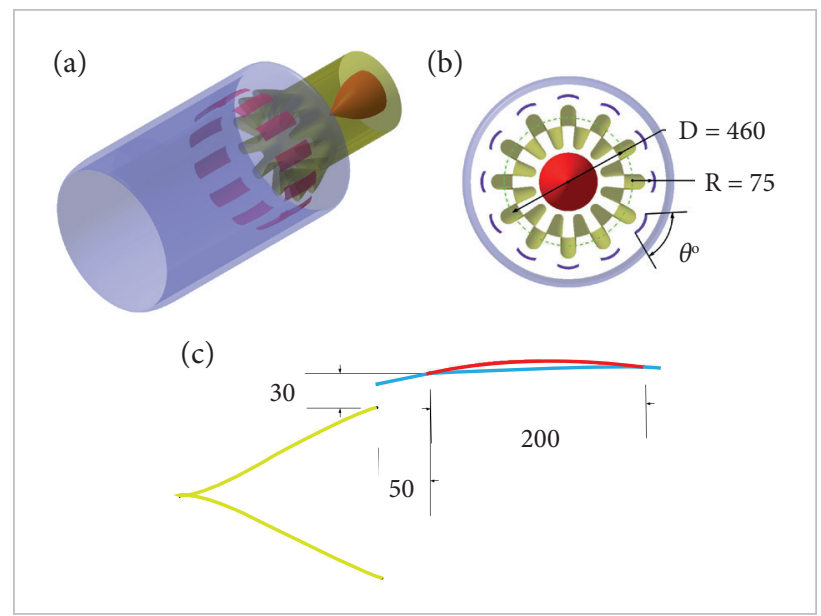

Figure 2. Geometrical dimensions of the spoilers lobe mixer. (a) Geometrical dimensions of the spoiler lobe mixer; (b) The geometrical dimensions for each spoiler.

\section{NUMERICAL SIMULATION METHOD}

The numerical simulation model is illustrated in Fig. 3 (Liu et al. 2015). Owing to the complex geometry, tetrahedral cells are used to discretize the simulation domain. Three-layer prism cells are used as the boundary cells with the first layer at the height of $0.05 \mathrm{~mm}$. As indicated by the arrow in Fig. 3, a refined domain is employed where drastic changes of the velocity and temperature occur. In order to accurately simulate the streamwise vortices, the maximum size of the cells in the refined domain is about $15 \mathrm{~mm}$. The number of cells in the refined domain is more than 20 million, and the total number of cells

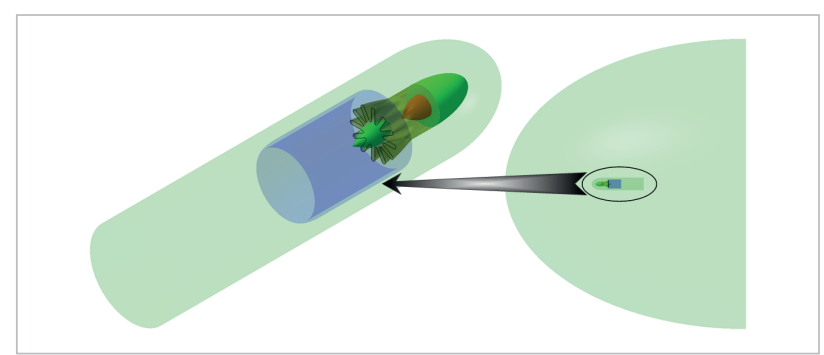

Figure 3. Numerical simulation model. 
in the whole simulation domain is more than 23 million. This mesh density has been validated to guarantee that the obtained temperature isosurface can trace out the mixing process well (Sheng et al. 2015a).

The flow-field simulation was based on Fluent software packages (ANSYS Inc). Three-dimensional Reynolds averaged $\mathrm{N}-\mathrm{S}$ equations were solved. The turbulence model is the SST $k$ - $\omega$, and the turbulence intensity was set to $5 \%$. The far-field boundary condition was set to velocity inlet and pressure outlet. The operating pressure was $101,325 \mathrm{~Pa}$, and the temperature was $300 \mathrm{~K}$. The discretized scheme was 2 nd-order. The jet inlet velocity was set to $125 \mathrm{~m} \cdot \mathrm{s}^{-1}$, and the temperature was $850 \mathrm{~K}$. In order to simulate the downwash, the secondary flow was also set to velocity inlet, which is $25 \mathrm{~m} \cdot \mathrm{s}^{-1}$. Energy equation was involved in the simulation. The same numerical simulation of jet mixing of lobed nozzle with the Fluent software packages has been used and validated in previous studies (Sheng et al. 2014; Sheng et al. 2015a; Sheng et al. 2015b; Liu et al. 2015).

In order to analyze the mixing of lobed nozzle more clearly, an illustration of the tailing-edge of the lobed nozzle and the downstream regions (Sheng et al. 2015b) is shown in Fig. 4. $\mathrm{A}$ is the lobe peak, B represents the sidewalls of the lobe and C is the lobe trough. For the downstream region of the lobed nozzle, the region in circle I is the core region; the region between I and II is that off the side walls; and the region off the lobe peaks is that between circles II and III.

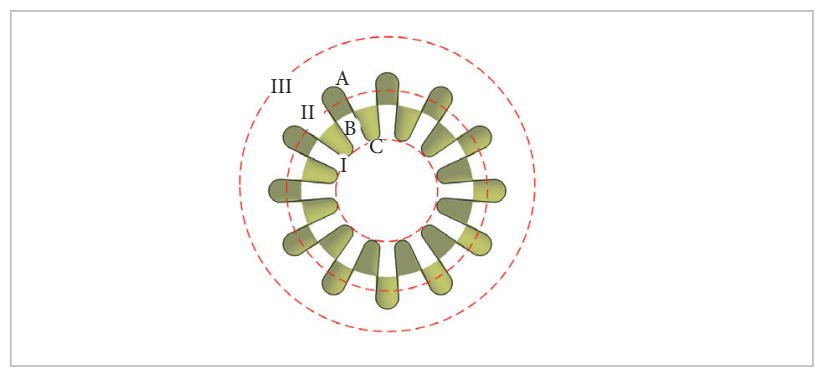

Figure 4. Illustration of the tailing-edge of the lobed nozzle and the downstream regions.

\section{JET MIXING PERFORMANCES FLOW RATIO}

In the present investigation, the flow ratio of primary and secondary streams was used to analyze the influence of spoilers on their mass flux. The flow ratio is defined as:

$$
\Phi=m_{\mathrm{s}} / m_{\mathrm{p}}
$$

where: $m_{s}$ and $m_{p}$ are the mass flux of the secondary and primary streams, respectively.

A larger flow ratio means more secondary stream can be supplied to the mixer. The flow ratio and relative variation of each scheme is given in Table 1 , where $\Delta \Phi$ is the relative variation of $\Phi$. Relative to the BLN scheme, the flow ratio of S1 and S2 is slightly increased, 0.29 and $0.039 \%$ improvements are achieved, while that of S3 decreased by $0.44 \%$. Thus, it can be concluded that, when the width of spoilers increased, the flow ratio decreased slightly, and the spoilers have little influence on flow ratio as the spoiler width in a certain range.

Table 1. Flow ratio and relative variation.

\begin{tabular}{|c|c|c|c|c|}
\hline & BLN & S1 & S2 & S3 \\
\hline$\Phi$ & 1.581998 & 1.586626 & 1.582615 & 1.575036 \\
\hline$\Delta \Phi / \%$ & 0.0000 & 0.2925 & 0.0390 & -0.4400 \\
\hline
\end{tabular}

\section{THE THERMAL MIXING EFFICIENCY}

The components of primary and secondary streams are treated as the same in the present investigation. The thermal mixing efficiency $\eta_{t r}$ (Xie and Liu 2011a) can be expressed as:

$$
\eta_{\mathrm{tr}}=1-\frac{\int\left(T_{\mathrm{m}}-T_{\mathrm{M}}\right)^{2} \mathrm{~d} m_{\mathrm{m}}}{T_{\mathrm{p}}^{2} m_{\mathrm{p}}+T_{\mathrm{s}}^{2} m_{\mathrm{s}}-T_{\mathrm{M}}^{2} m_{\mathrm{m}}}
$$

where: $m_{m}$ is the mass flux of the local mixing stream; the parameters $T_{p}$ and $T_{s}$ are the initial temperature of primary and secondary streams; $T_{m}$ is the temperature of the local mixing stream; $T_{M}$ is the temperature for the complete mixing stream of the primary and secondary streams, i.e.:

$$
T_{\mathrm{M}}=\frac{T_{\mathrm{p}} m_{\mathrm{p}}+T_{\mathrm{s}} m_{\mathrm{s}}}{m_{\mathrm{m}}}
$$

Thermal mixing efficiency indicates the degree of uniform mixing of the primary and secondary streams. It is a vital factor of the jet mixing. The thermal mixing efficiencies against $x / d$ is plotted in Fig. 5, where $x$ is the axial distance measured from the exit of the nozzle.

Figure 5 shows that the thermal mixing efficiencies for all the schemes increase rapidly from 0.25 to $1.0 \mathrm{~d}$ and then slow down. The values for the spoiler schemes are slightly lower than the BLN one in the range of $0.25-0.75 d$. This is because the spoilers were mounted in this axial position range of 
mixer tube, the primary and secondary streams impinged the spoilers, and the flow direction was changed. After $x=1.0 \mathrm{~d}$, the advantage of spoilers appears gradually, and the thermal mixing efficiencies for all the spoiler schemes are significantly higher than the BLN one. Smaller width spoilers can get a more significant enhancement.

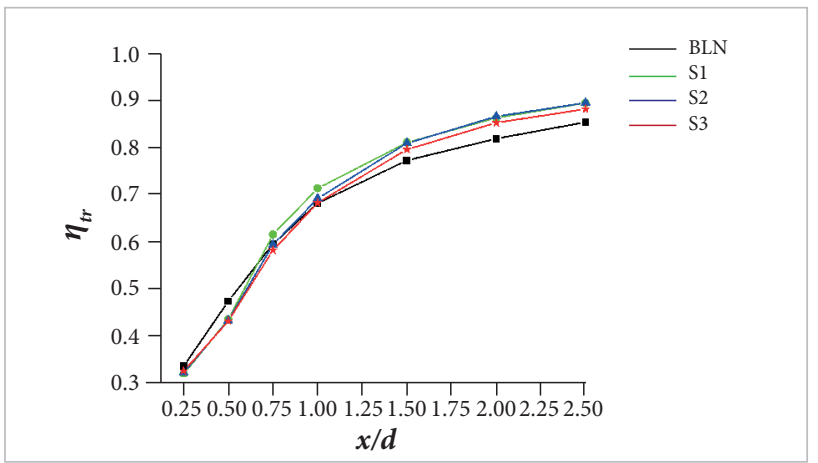

Figure 5. Thermal mixing efficiency along the axis for each lobed nozzle.

\section{THE TOTAL PRESSURE RECOVERY COEFFICIENT}

A superior total pressure recovery coefficient signifies less energy being consumed in the mixing. The equation is given by:

$$
\sigma=\frac{\int P_{\mathrm{m}}^{*} \mathrm{~d} m_{\mathrm{m}}}{\int P_{\mathrm{p}}^{*} \mathrm{~d} m_{\mathrm{p}}+\int P_{\mathrm{s}}^{*} \mathrm{~d} m_{\mathrm{s}}}
$$

where: $P_{m}^{\star}$ is the total pressure of the local mixing stream; the initial total pressure of primary and secondary streams is expressed, respectively, as $P_{p}^{*}$ and $P_{S}^{*}$.

It can be seen from Fig. 6 that the total pressure recovery coefficients of cross-sections along the lobed nozzles decreased

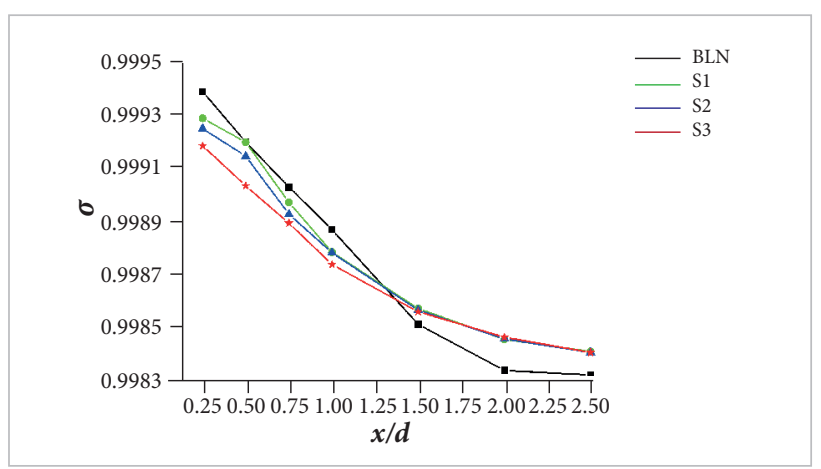

Figure 6. Total pressure recovery coefficients of crosssections along the lobed nozzles. rapidly up to $1.5 d$ and then slowed down. Because the primary and secondary streams impinged the spoilers, the coefficients of all spoiler schemes are lower than the BLN one between 0.25 and $1.0 \mathrm{~d}$. The influence of spoiler width is concreted before the cross-section $x=1.5 \mathrm{~d}$. For the spoiler schemes, the total pressure recovery coefficients decreased as the spoiler width increased, while, in the range between 1.5 and $2.5 d$, the spoiler schemes values were significantly higher than the BLN one. The spoiler width has little influence on the total pressure recovery coefficient value.

\section{THE STREAMWISE AND NORMAL VORTICITIES}

As the velocity ratio of primary and secondary streams is far greater than 1, the dominant factor of the mixing is the interaction between the streamwise and the normal vortices. Non-dimensional average vorticities of streamwise and normal vorticity were adopted to analyze the influence of spoiler. The non-dimensional streamwise average vorticities $\omega_{x}$ and the non-dimensional normal average vorticities $\omega_{n}$ are defined as:

$$
\omega_{x}=\frac{1}{m_{\mathrm{m}}} \int \frac{D}{u_{\mathrm{P}}}\left|\frac{\partial w}{\partial y}-\frac{\partial v}{\partial z}\right| \mathrm{d} m_{\mathrm{m}}
$$

$$
\omega_{n}=\frac{1}{m_{\mathrm{m}}} \int \frac{D}{u_{\mathrm{P}}} \sqrt{\left(\frac{\partial u}{\partial z}-\frac{\partial w}{\partial x}\right)^{2}+\left(\frac{\partial v}{\partial x}-\frac{\partial u}{\partial y}\right)^{2}} \mathrm{~d} m_{\mathrm{m}}(6)
$$

where: $D$ is the diameter of the mixing duct; $u_{P}$ is the initial velocity of the primary stream; $u, v$, and $w$ are the velocities in the $x, y$, and $z$ directions of the mixing stream, respectively.

Figures 7 and 8 are the non-dimensional average streamwise and normal vorticities along the axis of each alternating lobed

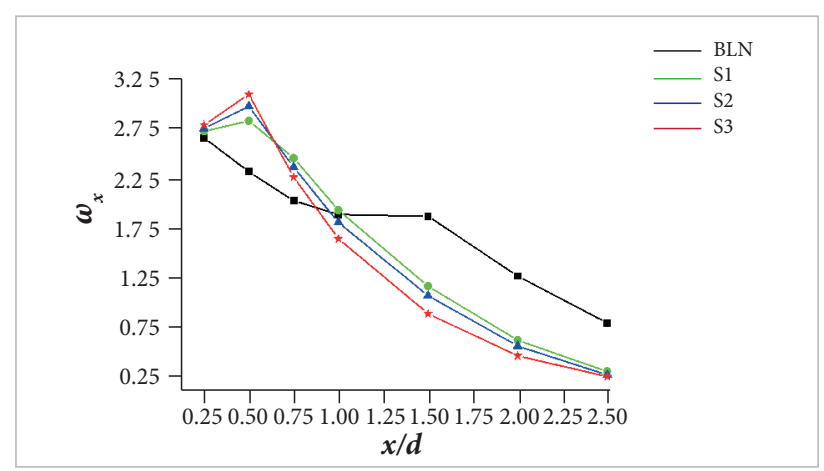

Figure 7. Non-dimensional average streamwise vorticities along the axis of each alternating lobed nozzle. 
nozzle. As can be seen in Fig. 7, in the mixing of the streamwise vorticities of the spoiler, schemes are increased between 0.25 and $0.5 d$ and then are slowed down quickly until the cross-section of $2.5 d$. For the BLN, the streamwise vorticities of cross-sections along the lobed nozzles decreased gently. The values of spoiler schemes were significantly higher than the BLN in the range between 0.25 and $1.0 \mathrm{~d}$. However, in the range of $1.0-2.5 d$, all of the spoiler schemes values were lower than those of the BLN one, and the streamwise vorticies decreased as the spoiler width increased. For the normal vorticities, as shown in Fig. 8, the trends were quite different for BLN and spoiler schemes. The normal vorticities of BLN have a significant first falling then rising and falling change process. For the spoiler schemes, the values of streamwise vorticies decreased gently in the whole mixing process. The spoiler width had a contrast influence in the 2 sides of the $1.0 \mathrm{~d}$ cross-section. The value increased between 0.25 and $1.0 d$ then decreased after the cross-section of $x=1.0 d$ as the spoiler width increased.

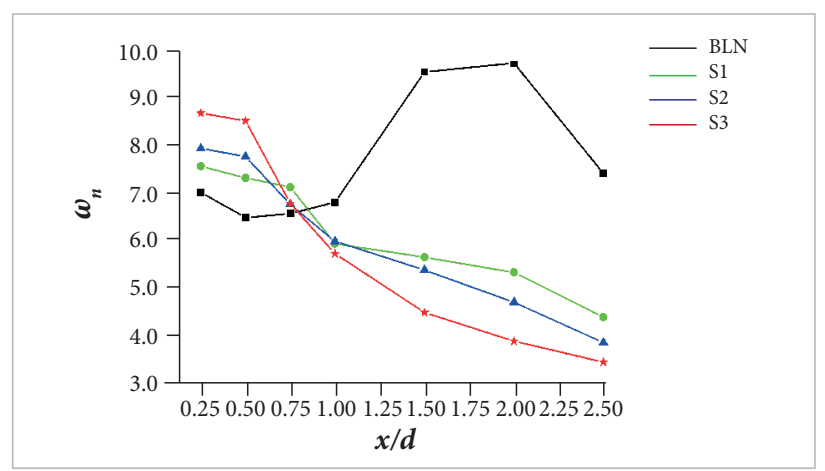

Figure 8. Non-dimensional average normal vorticities along the axis of each alternating lobed nozzle.

\section{JET MIXING FLOW-FIELD ANALYSIS}

Figure 9 shows the $700 \mathrm{~K}$ temperature isosurface of each scheme. As can be seen, for the BLN scheme, the mixing off the sidewalls region is slightly faster than the region off the lobed peaks. The complete mixing for the core region primary stream requires a long distance. The primary flow impinged the sidewalls of the mixer tube, while, for the spoiler schemes, the spoilers avoided the sidewalls squeezing phenomenon; the mixing in the regions off the lobed peaks and sidewalls was faster than that of the BLN scheme. As the widths of spoilers increased, the mixing rate in the regions off the sidewalls increased, while the mixing in the regions off the lobed peaks decreased.
Figure 10 shows distributions of the velocity vector, temperature, and axial velocity $\left(\mathrm{m} \cdot \mathrm{s}^{-1}\right)$ at 0.5 and $1.0 \mathrm{~d}$ for each scheme. The color of the velocity vector represents the distributions of temperature, and the velocity vectors scaling factors are maintained constant.

For the BLN scheme, in the region off the lobed peaks, the primary stream flowed outward and turned toward the two sides of lobed peaks. A pair of large intensive streamwise vortices was formed in the lobed peak region. The normal vortices are formed at the transition layer by the gradient of the axial velocity between the primary and secondary streams, while, in the cross-section $x=1.0 \mathrm{~d}$, the high-temperature primary stream flowing outward had reached and impinged the walls of the mixer tube. Strong normal vortices were formed because of the large velocity gradient. A high-temperature region in the walls of the mixer tube appeared. Meanwhile, in the region off the sidewalls, the secondary stream did not mix adequately, and a distinct lowtemperature region existed.

The spoilers significantly changed the primary and secondary streams flowing in the region off the lobe peaks. Part of the hightemperature primary stream flipped up along the two ends of the spoiler, meanwhile some of the primary streams flipped down. Two pairs of significant streamwise vortices were formed in the upper and lower sides along the two ends of each spoiler.

We can see in Fig. 10 that, as the widths of the spoiler increased, the mixing in the region off the sidewalls accelerated, while the mixing region in the region off the lobe peaks decelerated. This is due to the fact that: as the width increased, the degree of arc for the spoiler enlarged ( $\mathrm{S} 1$ is $40^{\circ}, \mathrm{S} 2$ is $60^{\circ}$ and

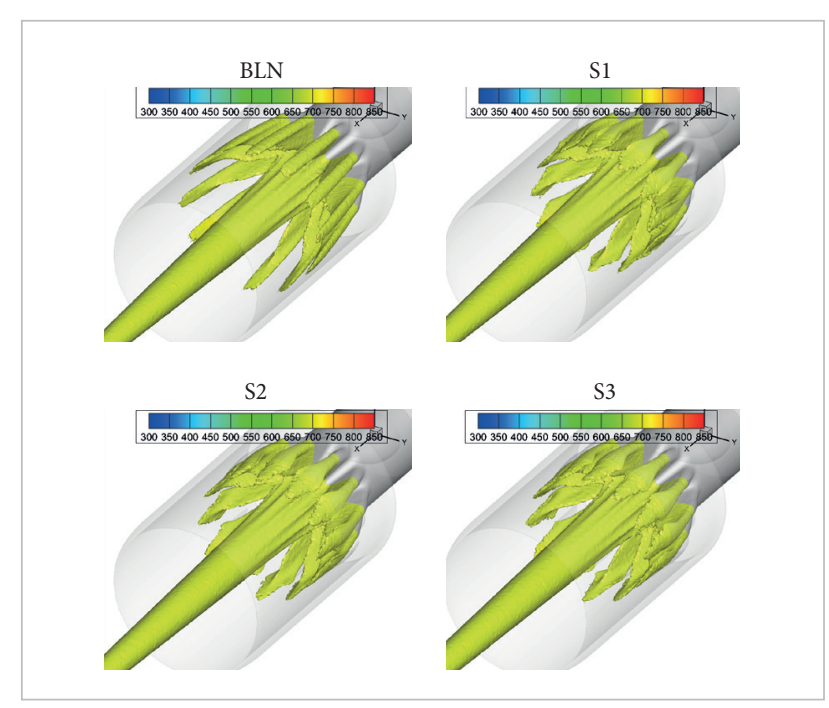

Figure 9. The 700-K temperature isosurface of each scheme. 
$\mathrm{S} 3$ is $90^{\circ}$ ), then the spoiler forced more parts of primary stream flipping down and less parts of flipping up. Meanwhile, the two ends of the spoiler have a longer distance from the sidewalls to the mixer tube. The pair of streamwise vortices on the upper sides of the spoilers is reduced and has a longer distance from the sidewalls of the mixer tube. More secondary streams did not mix effectively, and the mixing in the region off the lobe peak decreased. However, the pair of the streamwise vortices on the lower sides of the spoilers increased, and the interaction between the streamwise vortices induced by the adjacent spoilers enhanced. So the mixing in the region off the sidewalls accelerated.

Figure 11 shows the temperature contours at $2.5 d$ of each lobed nozzle. The temperature had a significant difference
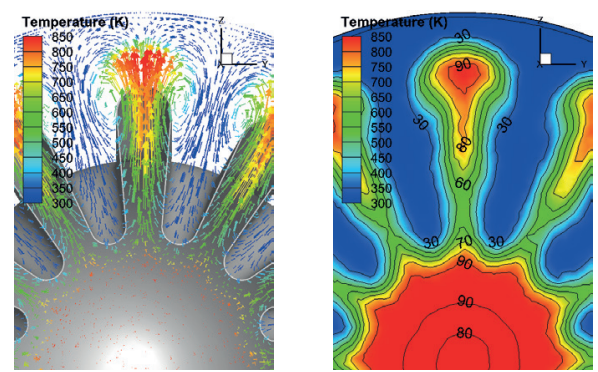

(a) BLN $-0.5 d$ cross-section
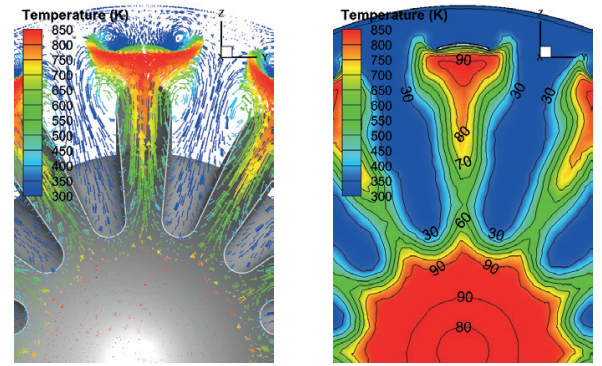

(c) $\mathrm{S} 1-0.5 d$ cross-section
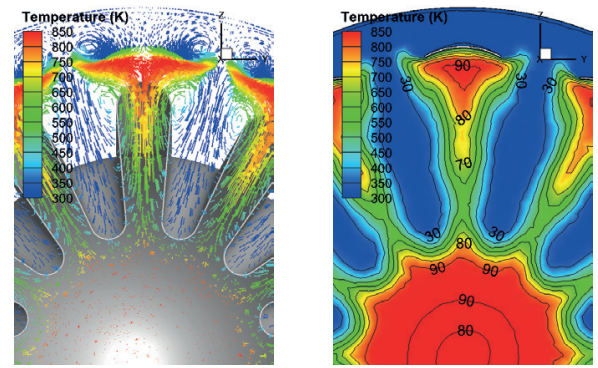

(e) $\mathrm{S} 2-0.5 d$ cross-section
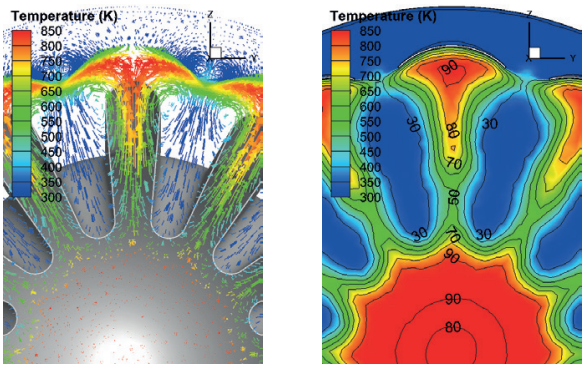

(g) S3 - $0.5 d$ cross-section
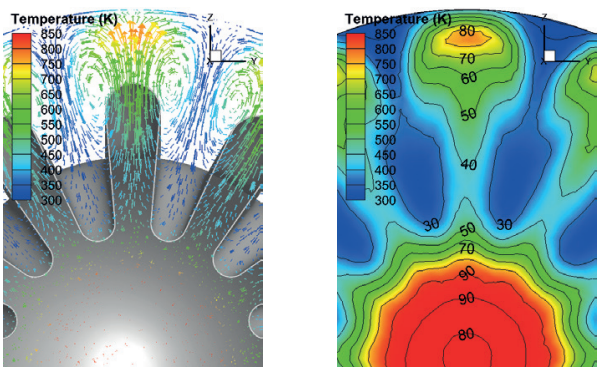

(b) BLN - $1.0 d$ cross-section
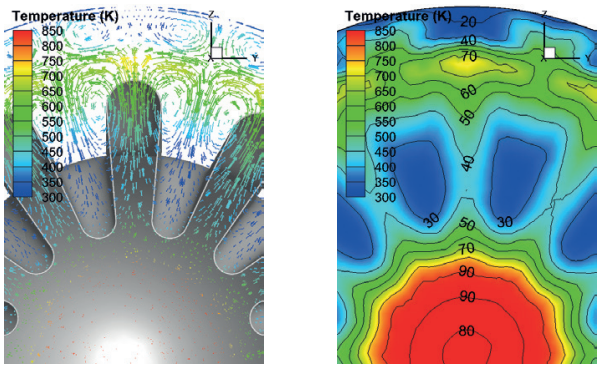

(d) $\mathrm{S} 1-1.0 d$ cross-section
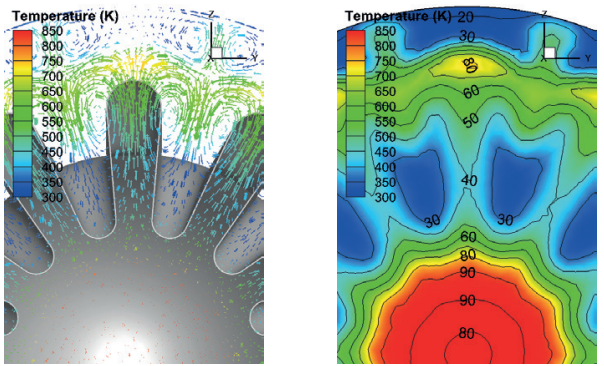

(f) $\mathrm{S} 2-1.0 d$ cross-section
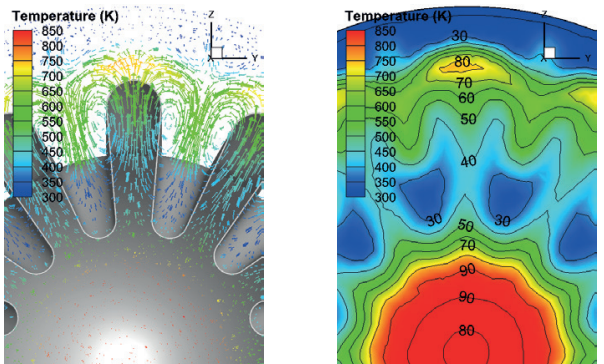

(h) S3 - $1.0 d$ cross-section

Figure 10. Distributions of the velocity vector, temperature and axial velocity $\left(\mathrm{m} \cdot \mathrm{s}^{-1}\right)$ at $0.5 d$ and $1.0 d$ for each scheme. 


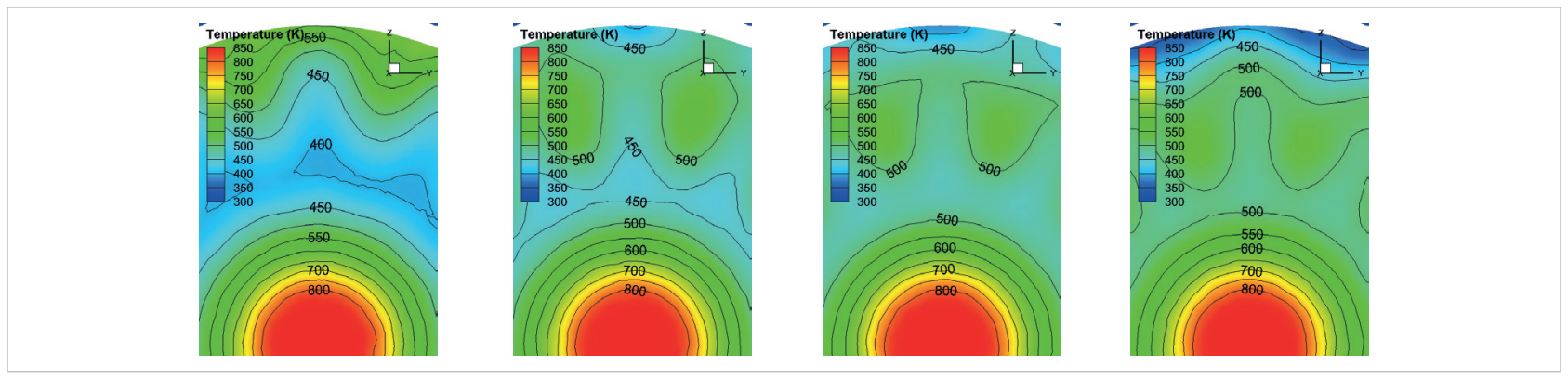

Figure 11. Temperature contours at $2.5 d$ of each lobed nozzle.

between the BLN and spoiler schemes. For the BLN scheme, a distinct low-temperature region existed between the sidewalls of the mixer tube and the primary core region; the temperature of the mixer tube sidewalls was relatively high. The secondary stream mixing was inadequate, while, for the spoiler schemes, the spoilers changed the flow direction, the mixing of primary and secondary streams was more sufficient, and the low-temperature contours region appeared near the mixer tube sidewalls. As the width of spoilers increased, the temperature of the mixer sidewalls was distributed more uniformly.

\section{CONCLUSIONS}

In the present study, spoilers with different widths were mounted on the lobed nozzle. The effect of the spoilers on the jet mixing and its mechanism were analyzed by numerical method. The results show that the flow ratio of primary and secondary flows almost did not change with or without the spoilers, which increased the borderline area of primary and secondary flows; besides, the mixing was accelerated. The spoilers avoided that the primary flow impinged the sidewall, and the temperature of the mixing tube wall decreased significantly. There were significant improvements for the total pressure recovery coefficient and the thermal mixing efficiency after the $x=1.5 d$ cross-section. As the spoiler width increased, the mixing rate in the region off the sidewalls increased, while the mixing in the region off the lobe peaks decreased.

\section{AUTHOR'S CONTRIBUTION}

Dawei L, Jun H, Zhiqiang S and Jinzu J conceived the idea; Dawei L and Zhiqiang S wrote the main text; Jun $\mathrm{H}$ and Jinzu J gave the main guide for the simulation and paper writing; Dawei L and Zhiqiang S performed the simulation; author Zhiqiang S prepared the figures. All authors discussed the results and commented on the manuscript.

\section{REFERENCES}

Acheson KE, Marques EC, Moore MD (2011) A partial mixer nozzle for turbofan nacelles to provide significant jet mixing control. AIAA11-3189. Proceedings of the 29th AIAA Applied Aerodynamics Conference; Honolulu, USA.

Elliott JK, Manning TA, Qiu YJ, Greitzer, EM, Tan CS, Tillman TG (1992) Computational and experimental studies of flow in multi-lobed forced mixers. AIAA-92-3568. Proceedings of the 28th Joint Propulsion Conference and Exhibit; Nashville, USA.

Liu DW, Huang J, Sheng ZQ, Ji JZ (2015) The effects of scalloping width and position on jet mixing of lobed nozzles. J Aerosp Technol Manag 7(4):425-431. doi:10.5028/jatm.v7i4.519

McCormick DC, Bennett JC (1994) Vortical and turbulent structure of a lobed mixer free shear layer. AIAA J 32(9):1852-1859. doi: $10.2514 / 3.12183$

O’Sullivan MN, Krasnodebski JK, Waitz IA, Greitzer EM, Tan CS, Dawes
WN (1996) Computational study of viscous effects on lobed mixer flow features and performance. J Propul Power 12(3):449-456. doi: $10.2514 / 3.24056$

Pan CX, Zhang JZ, Ren LF, Shan Y (2014) Effects of rotor downwash on exhaust plume flow and helicopter infrared signature. Appl Therm Eng 65(1-2):135-149. doi: 10.1016/j.applthermaleng.2014.01.009

Pan CX, Zhang JZ, Shan Y (2013a) Effects of exhaust temperature on helicopter infrared signature. Appl Therm Eng 51(1-2):529-538. doi: 10.1016/j.applthermaleng.2012.09.016

Pan CX, Zhang JZ, Shan Y, Wang XW (2013b) Flow separation control of lobed ejector/forced mixer by ventilation. J Aero Power 28(2):331-337. In Chinese.

Povinelli LA, Anderson BH (1984) Investigation of mixing in a turbofan exhaust duct, part II: computer code application and verification. AIAA ل 22(4):518-525. doi: 10.2514/3.8433 
Presz Jr WM, Reynolds Jr G, McCormick D (1994) Thrust augmentation using mixer-ejector-diffuser systems. AIAA-94-0020. Proceedings of the 32nd Aerospace Sciences Meeting and Exhibit: Reno, USA.

Shan Y, Zhang JZ (2005) Numerical investigation on the effects of lobe nozzles geometric parameters on mixer-ejector performance. J Aero Power 20(6):973-977. In Chinese.

Sheng ZQ, Chen SC, Wu Z, Huang PL (2015a) High mixing effectiveness lobed nozzles and mixing mechanisms. Sci China Technol Sci 58(7):1218-1233. doi: 10.1007/s11431-015-5846-8

Sheng ZQ, Huang PL, Ji JZ, Wang Y (2014) Effects of modification on jet mixing of sword alternating lobed nozzle. J Beijing Univer Aeronau Astronau 40(10):1417-1423. doi: 10.13700/j.bh.1001 5965.2013.0633
Sheng ZQ, Huang PL, Zhao T, Ji JZ (2015b) Configurations of lobed nozzles for high mixing effectiveness. Inernational Journal of Heat and Mass Transfer 91:671-683. doi: 10.1016/j. ijheatmasstransfer.2015.08.022

Shumpert PK (1980) An experimental model investigation of turbofan engine internal exhaust gas mixer configurations. In Proceedings of the 18th AIAA Aerospace Sciences Meeting; Pasadena, USA.

Xie Y, Liu YH (2011a) A modified thermal mixing efficiency and its application to lobed mixer nozzle for aero-engines. Heat Transf Res 42(4):317-335. doi: 10.1615/HeatTransRes.2011003398

Xie Y, Liu YH (2011b) Effects of lobe penetration ratio on performance of mixer exhaust system. J Propul Tech 32(2):207-213, 252. In Chinese. 